\title{
Repetition priming in simple addition depends on surface form and typicality
}

\author{
SONIA C. SCIAMA \\ University College London, London, England \\ CARLO SEMENZA \\ University of Trieste, Trieste, Italy \\ and \\ BRIAN BUTTERWORTH \\ University College London, London, England
}

\begin{abstract}
Repetition priming and recognition memory for numbers were measured in four experiments using single-digit addition. Results of the first two experiments indicate that when numbers were presented as number words and dot configurations, preexposure of the same problem in the same notation produced greater reaction-time benefit than did preexposure of the same problem in Arabic-digit notation. In contrast, when numbers were presented as Arabic digits, preexposure of the same problem in Arabic digit, number word, and dot notation produced the same amount of priming. In the third experiment, priming was shown to be greatest, for all three notations, when the task performed on preexposure trials (addition or multiplication) matched the task performed on repetition trials (addition). Results of the fourth experiment, measuring recognition memory, were comparable to the priming results in the sense that memory was superior when notation matched across repetitions if the test involved number words and dot configurations but not Arabic digits. These data are interpreted in terms of models of numerical cognition, and they support the hypothesis that the influence of surface form on repetition priming depends on the typicality of the input for the task.
\end{abstract}

Numbers can be represented in many different ways. They can be represented by Arabic digits and Roman numerals, as well as by other numeration systems that did not reach Western culture. In some cases, notations were specifically developed for calculation. The Arabic notation, for example, with its place-value system is particularly well suited for both written and mental calculation (Zhang \& Norman, 1995). Even in single-digit computations, different notations may have different consequences for mental operations. Analogical systems may encourage counting procedures, whereas other notations may result in retrieval of memorized number facts (e.g., $5+5=10$ ). The organization of the system of number facts may itself depend on the structure of a particular numeration system. The aim of the present experiments was to examine the influence of notation on the representation of numerical information.

The effect of performing an arithmetical operation presented in one notation on the subsequent performance of

This article is based on work carried out at the University of Padua, Italy, supported by European Union Grant EU 904/00/480, and at University College London, supported by Wellcome Trust Grant 045013 . The authors thank Sara Mondini and Monica Morello for help collecting the data. The authors also thank Michael McCloskey for comments on an earlier version of the paper. Correspondence should be addressed to S. C. Sciama, Department of Psychology, University College London, Gower Street, London WC1E 6BT, England (e-mail: s.sciama@ucl.ac.uk). the same operation presented in another notation was measured in a repetition priming paradigm in which prime sums and target sums were presented in two blocks (study and test) separated by an intervening task. If different notations access a common representation, then performance will be facilitated when the same sum is repeated in different notations. Similar reasoning has been used in studies examining the effects of extended practice (Rickard \& Bourne, 1996; Rickard, Healy, \& Bourne, 1994).

Although repetition priming is a well-established effect in word and object recognition tasks (Schacter, Chiu, \& Ochsner, 1993), it has not received much attention in research on numerical tasks (except Arbuthnott, 1996, who studied the effects of immediate repetition). Simple singledigit arithmetical problems, such as $5 \times 3$ or $5+3$, could be performed by a counting procedure (Groen \& Parkman, 1972), but there is strong evidence that, for small problems, the answer is simply retrieved from memory (Ashcraft, 1992; LeFevre, Sadesky, \& Bisanz, 1996). In some conditions, it may even be retrieved automatically (LeFevre, Bisanz, \& Mrkonjic, 1988; Zbrodoff \& Logan, 1986). Thus, the solution of simple arithmetical problems should be influenced by repetition, because preexposure to a problem would be expected to facilitate retrieval of the answer on a subsequent occasion.

Kolers (1973) studied the effects of typography on facilitation in encoding and recognition of sentences and concluded that the operations involved in analyzing the 
visual array of letters was retained in memory and was tied to the conceptual record of the event. Subsequent studies further investigating memory for perceptual surface form have yielded a debate as to whether effects of the visual array result from atypical or transformed typography (Brown \& Carr, 1993; Jacoby \& Hayman, 1987; Jacoby, Levy, \& Steinbach, 1992). Various studies have shown that the perceptual information contained in handwriting has more influence than does the perceptual information contained in standardized type (Brown \& Carr, 1993; Roediger \& Blaxton, 1987). In one set of experiments, for example, timed reading of handwritten, but not typed, words benefited more from prior exposure to the same words printed in the same typography than from prior exposure to the same words printed in a different typography (Brown \& Carr, 1993). In the numerical domain, the Arabic notation has become the standard for calculation purposes, and other systems, such as number words (e.g., five) and dot configurations - for example,

$$
\because:
$$

-are less typical. Thus, surface-form effects might depend on notation in a calculation task.

Research on the representation of number has tried to determine whether number notation has an impact only at initial encoding stages or whether it has consequences for later stages in processing (Gonzalez \& Kolers, 1982; Noel \& Seron, 1992). According to the abstract-modular model of number processing (McCloskey, Caramazza, \& Basili, 1985), there is a common abstract code into which different notations are translated, and the encoding phase is independent of later stages of elaboration, such as calculation. It consists of four components: comprehension (or encoding) systems, an abstract representation system, calculation mechanisms, and production systems. Representation of arithmetical facts does not depend on any existing notation, and it is isolated from syntactic comprehension systems. A very similar model is the identicalelements model (Rickard \& Bourne, 1996; Rickard et al., 1994), in which there are distinct and sequential perceptual, cognitive, and motor stages of performance. In both models, any differences in responses to different number notations for the same task, such as different types of errors (Campbell, 1994), result from differences in the encoding procedures.

A second approach is described by the triple-code model (Dehaene, 1992; Dehaene \& Cohen, 1995), in which there are three types of mental code for numbers: a visual Arabic code, an auditory-verbal code, and an analogical quantity representation. In this model, the input notation is translated into a particular code appropriate for the task to be performed. If the task is magnitude comparison, the input is translated into a quantity representation; if the task is a simple arithmetical operation, the input is translated into an auditory-verbal code, and the response is retrieved from memory. The model includes an anatomical implementation in which the vi- sual Arabic number code and the magnitude representation are processed in both hemispheres, but the auditory-verbal code is processed only in the left hemisphere. This model differs from the abstract-modular model and the identicalelements model because it postulates multiple perceptually based number codes, with each code specific to a set of tasks. However, they share the assumption that arithmetical number facts are stored in memory in a single (in this case, auditory-verbal) code that is isolated from the encoding system. The input notation becomes irrelevant once it is translated into the auditory-verbal code.

A third model is the preferred-entry code model, according to which each individual has a preferred mental code that is based on either Arabic digits or number words (Noel \& Seron, 1993). The input is translated into the preferred code on which later processes operate. Again, the input notation is irrelevant once it is translated into the preferred code. The three models described above will be referred to as common-code models, because there is a single code into which different inputs are translated.

An approach distinct from the common-code models is Campbell's encoding complex hypothesis, which postulates that different number notations result in different mental codes that are tied to the input notation (Clark \& Campbell, 1991). The encoding complex hypothesis maintains that, even for the same task, different symbolic notations result in different mental processes beyond an encoding stage. Semantic memory for number facts is assumed to consist of a network of interconnected representations expressed in terms of codes that correspond to Arabic digits and number words. These codes may be connected via a magnitude representation (Campbell, 1995). The hypothesis is motivated as an alternative to the abstract-modular model (Campbell, 1994), and it deemphasises the role of abstract representation while emphasizing the role of format-specific processes. Thus, the approach does not make specific predictions; however, as Campbell (1994) stated "different notations or surface forms for numbers can directly influence the strategies, processes, or codes that contribute to given tasks" (p.4).

The experiments reported here tested the limits of this claim, investigating both the place of a common code and the influence of notation. An alternative proposal is suggested in which both a common code and form-specific codes coexist. This corresponds to a general approach to mental representation that acknowledges a role for both abstract and form-specific representation (Brown \& Carr, 1993; Marsolek, Kosslyn, \& Squire, 1992).

Evidence for an influence of surface form beyond encoding processes has been found in on-line performance of arithmetical tasks. In one study, presentation of one addend followed by a second addend, instead of both addends simultaneously, had different consequences for different notations and different operations (Blankenburger $\&$ Vorberg, 1997). Addition performed on a number-word notation was substantially facilitated by this procedure. Another demonstration has shown that the particular structure of a notation can influence performance in verifica- 


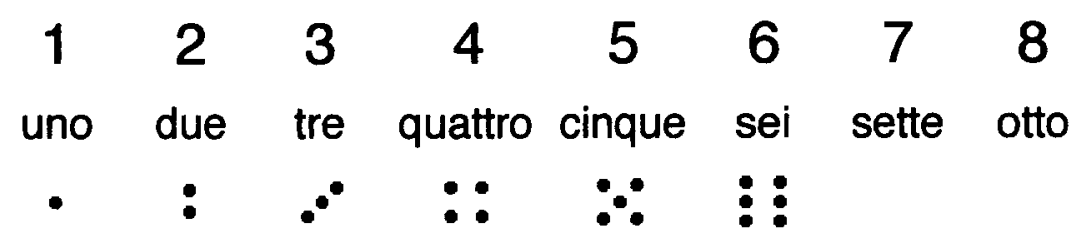

Figure 1. The three number notations used in the experiments. Italian words were used with Italian subjects.

tion and production of simple arithmetical facts (Noel \& Seron, 1997). For example, equation verification with mixed-notation equations (e.g., IV $=5-1$, or IV $=2+$ 2) was faster when the operation required to encode the Roman numeral mimicked the operation required to produce the solution to the problem expressed in Arabic digit notation (e.g., IV $=5-1$, and not IV $=2+2$ ).

The experiments reported below suggest that an influence of notation on repetition priming in simple addition results from the representation of form-specific information. These representations do not contribute to independent encoding processes as suggested by common-code models, but they facilitate performance for particular notations by representing the addition information in a way that is specific to the input notation. They differ from the representations postulated by the encoding complex hypothesis because they are not assumed to constitute the stable semantic memory system.

The first two experiments investigated repetition priming in simple addition both to establish its presence and to measure the effect of changing number notation across repetitions. In Experiment 1, numbers were presented as Arabic digits and number words; in Experiment 2, they were presented as Arabic digits and dot configurations (see Figure 1). If the processes involved in performing simple addition are completely determined by the input notation, there should be no priming when notation is changed across repetitions. In contrast, equal priming across notations would suggest that the representations and processes for different notations are the same, as postulated by the common-code models. The intermediate situation in which priming is simply diminished when the notation is changed across repetitions would indicate that, as well as sharing common processes, the different input notations are also represented differently. Following results for verbal tasks (Brown \& Carr, 1993), surface-form effects would be expected when addition is tested with atypical notations, such as number words and dot configurations (i.e., notations not typically used in addition).

A third experiment was performed to determine whether surface-form effects could be attributed to facilitation of encoding processes as suggested by commoncode models. In these models, encoding procedures do not depend on the arithmetical operation; therefore, if priming reflects facilitated encoding, then it should occur when the same numbers are presented in the same notation but in different operations. Experiment 4 tested the possibility that atypical notations have consequences for recognition memory. If addition performed on an atypical notation is represented in memory as a distinctive event, this could lead to higher levels of recognition memory when the distinctive information is used as a retrieval cue. This experiment also tested the possibility that any priming found in Experiments 1 and 2 might depend on conscious retrieval processes.

\section{EXPERIMENT 1}

In Experiment 1, numbers were presented as Arabic digits or number words. One third of the problems were preexposed in the same notation, one third in a different notation, and one third were not preexposed. If addition performed on Arabic digits and number words occurs by retrieval of an addition fact represented in a common code, then priming should transfer across these notations. Number-word notation might result in processes absent for Arabic digits, because it is not the notation normally used in calculation. This could lead to distinctive representation in terms of these processes on the first presentation, and to retrieval that depends on surface form on the second presentation, and, therefore, to benefit gained from repetition of the problem in the same notation.

\section{Method}

Subjects. Forty-eight undergraduates at the University of Padova participated as subjects. They were native Italian speakers.

Materials. The experiment was controlled by a Macintosh SI computer. Responses were detected using a voice-activated relay.

The stimuli consisted of three sets of five pairs of single-digit numbers (see Appendix A). The number pairs served as the addends to be summed, and each set was assigned to one of the three study conditions (match, mismatch, new). The sets contained pairs of numbers that added to $7,8,9$, and 10 ; two sets contained a pair that added to 5 , and one set contained a pair that added to 4 . In this way, the delay of the voice-activated relay caused by the initial sound uttered was balanced across conditions, because responses in the different conditions would begin with the same letters. The sets were also balanced so that the total of all the larger addends and the total of all the smaller addends for each set differed by not more than 2 . The assignment of sums to conditions was counterbalanced across subjects so that each sum appeared in each study condition eight times.

Numbers were presented one above the other in the center of the screen with no addition sign, as shown in Figure 2.

A list of 60 concrete nouns, four to seven letters long, was used in an intervening task.

Design and Procedure. The subjects were randomly assigned to a test notation group. For half the subjects, the test word group, the 

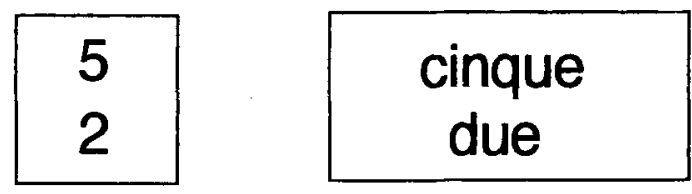

Figure 2. Examples of the stimuli used in Experiment 1.

test items appeared as words; for the other half, the test digit group, the test items appeared as Arabic digits. Within each of these groups, study items appeared either as number words or as Arabic digits. Each set of sums was assigned to one of three study conditions (match, mismatch, new). Sums were preexposed at study in the test notation (match) or in the alternative notation (mismatch), or they were not preexposed (new). All sums were presented both with the larger addend above the smaller addend and with the smaller addend above the larger addend in separate blocks. This was done solely to increase the number of observations.

Each subject received a different random ordering of the sums, with the constraint that every three (or two for the study phases) sums there was a representative of each study condition.

A session lasted approximately $20 \mathrm{~min}$ and consisted of four phases. There were two study phases in which sums were preexposed (the primes) and two test phases in which sums were repeated (the targets): Study A, Test A, Study B, and Test B. Sums presented as primes in each study phase were repeated as targets in the subsequent test phase. In Study $\mathrm{A}$ and Test $\mathrm{A}$, the larger addend appeared above the smaller addend; in Study B and Test B, the smaller addend appeared above the larger addend. Half the subjects received Study A and Test A first, followed by Study B and Test B; the other half received Study B and Test B, followed by Study A and Test $\mathrm{A}$. The task in all these phases of the experiment was to sum the numbers as quickly and accurately as possible. Between each of these phases, there was an intervening task in which a list of 20 words was presented to be read aloud by the subject. Words were exposed until a vocal response was detected, and there was an interstimulus interval of $1,500 \mathrm{msec}$. At the end of each task, a message appeared on the monitor informing the subject that the task was over, and the experimenter then reminded the subject of the task to be performed next. Each addition phase began with a buffer sum for which reaction time (RT) was not recorded.

Individual addition trials consisted of the following events: a rectangle was displayed for $500 \mathrm{msec}$; the numbers then appeared inside the rectangle. The display was cleared when the subject responded. After the experimenter entered the response on the computer keyboard, the next trial began. The subjects received four practice trials before starting.

\section{Results and Discussion}

Incorrect responses and responses with latencies more than 2.5 standard deviations ( $S D \mathrm{~s}$ ) from the mean of each subject were removed from the analysis. This resulted in elimination of $5 \%$ (errors, $2 \%$; outliers, $3 \%$ ) of the total number of responses for the subjects in the test digit group and $8 \%$ (errors, $5 \%$; outliers, $3 \%$ ) for the subjects in the test word group. For each subject, the outliers were replaced by their mean plus 2.5 times their $S D$.

The results are shown in Table 1. A 2 (test notation: word, digit) $\times 3$ (study: match, mismatch, new) $\times 2($ addend order: larger addend above, larger addend below) mixed-design analysis of variance (ANOVA) was performed on mean RTs, with test notation as a betweensubjects factor. There was a main effect of test notation
$\left[F(1,46)=67.47, M S_{\mathrm{e}}=88,960.73\right],{ }^{1}$ indicating that numbers presented as words took longer to sum than did numbers presented as Arabic digits (words, $986 \mathrm{msec}$; digits, $697 \mathrm{msec}$ ). There was a main effect of addend order $\left[F(1,46)=13.15, M S_{\mathrm{e}}=8,357.91\right]$; trials in which the larger number was presented below the smaller number took longer to sum $(861 \mathrm{msec})$ than did trials in which the larger number was presented above the smaller number $(822 \mathrm{msec})$. This variable did not, however, interact with any other variable and will not be discussed further because it was included only to increase the number of observations. Finally, there was a main effect of study $\left[F(2,92)=15.04, M S_{\mathrm{e}}=8,019.02\right]$. As can be observed in Table 1, latencies for new sums were longer than latencies for repeated sums.

The interaction between test notation and study was marginally significant $\left[F(2,92)=2.49, M S_{\mathrm{e}}=8,019.02\right.$, $p=.09]$. To further investigate the effect of study, planned comparisons were performed separately for each test notation group. For the test word group, all study conditions were found to differ significantly [match vs. mismatch, $F(1,23)=8.42, M S_{\mathrm{e}}=4,055.97$; match vs. new, $F(1,23)=11.60, M S_{\mathrm{e}}=19,116.07 ;$ mismatch vs. new, $\left.F(1,23)=4.15, M S_{\mathrm{e}}=19,717.71\right]$, whereas for the test digit group, there was no significant difference between the match and mismatch conditions [match vs. mismatch, $F(1,23)=0.10, M S_{\mathrm{e}}=2,066.12$; match vs. new, $F(1,23)=$ $17.70, M S_{\mathrm{e}}=2,205.32$; mismatch vs. new, $F(1,23)=$ 47.03, $\left.M S_{\mathrm{e}}=952.92\right]$. Thus, only the subjects tested with number words demonstrated a surface-form effect.

The presence of priming across Arabic digits and number words is predicted by the common-code models. However, a role for notation-specific representation was also demonstrated by the surface-form effect for the test word group. This effect could have resulted either from facilitated encoding for number words or from representation of addition information that retained details of surface form.

Similar results were found by Rickard and Bourne (1996), who measured transfer of practice across Arabicdigit and number-word notations after repeated presentations in a multiplication task. Like the present results, practice transferred across notations, and Rickard and Bourne took this as evidence for a common semantic code representing arithmetical information. They did, however, find surface-form effects for both Arabic digits and num-

Table 1

Mean Reaction Times and Their Standard Errors (in Milliseconds) and Error Proportions as a Function of Test Notation Group and Study in Experiment 1

\begin{tabular}{|c|c|c|c|c|c|c|}
\hline \multirow[b]{4}{*}{ Study } & \multicolumn{6}{|c|}{ Test Notation Group } \\
\hline & \multicolumn{3}{|c|}{ Digit } & \multicolumn{3}{|c|}{ Word } \\
\hline & \multicolumn{2}{|c|}{ RT } & \multirow[b]{2}{*}{ Errors } & \multicolumn{2}{|c|}{ RT } & \multirow[b]{2}{*}{ Errors } \\
\hline & $M$ & $S E$ & & $M$ & $S E$ & \\
\hline Match & 684 & 18.09 & .02 & 941 & 22.51 & .03 \\
\hline Mismatch & 682 & 18.22 & .02 & 979 & 20.18 & .06 \\
\hline New & 725 & 18.09 & .03 & 1,037 & 26.38 & .06 \\
\hline
\end{tabular}




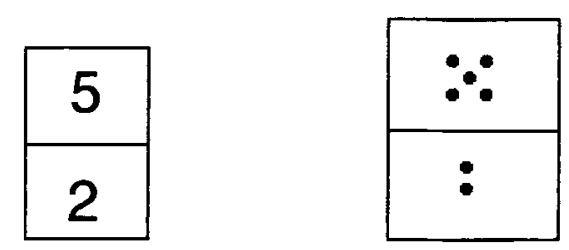

Figure 3. Examples of the stimuli used in Experiment 2.

ber words, but, in agreement with the results found here, the effect was larger for number words. There are several differences between the methodology used here and the methodology used by Rickard and Bourne: They used multiple repetitions (in two sessions), whereas we used a single repetition; they used multiplication problems with double-digit answers, whereas we used addition problems with answers not exceeding 10; and their subjects responded manually on a keyboard, whereas our subjects gave a spoken response. Any one of these factors might have resulted in a surface-form effect for Arabic digits. When they divided their problems into easy and difficult sets, they found a trend for the problem difficulty $\times$ type of repetition (match vs. mismatch) interaction, and this interaction seems to be present only for Arabic digits. For the Arabic-digit notation, more difficult problems produced a larger surface-form effect than did easier problems. Thus, despite their differences, the results reported here and those of Rickard and Bourne converge. Both suggest that the influence of prime notation is affected by typicality.

Experiment 2 was performed to investigate the processing of a third notation for numbers: dot configurations. Like number words, dot configurations are not normally used to denote numbers for the purposes of calculation. If surface-form effects depend on typicality, then dot configurations should show results similar to those for number words.

\section{EXPERIMENT 2}

Experiment 2 was similar to Experiment 1 except that numbers were presented either as Arabic digits or as the dot configurations that appear on a die (see Figure 1). In Experiment 1, a surface-form effect was only present for number words. It is possible that Arabic digits showed no sensitivity to the notation of the first presentation because Arabic digits and number words, both being discrete, are not sufficiently different from each other. Dot configurations, which are analogical, might provide the conditions necessary to produce a surface-form effect when compared with a discrete system, such as Arabic digits.

A secondary goal of Experiment 2 was to exclude the possibility that the priming effects of Experiment 1 reflected consciously directed memory strategies (Jacoby, 1991). Subjects might respond to the repeated addition problems on the basis of directed recollection of the first presentation. This is highly improbable because recol- lection is likely to have been more effortful than the addition task itself. Nevertheless, in order to test the potential effects of such strategies, one group of subjects was required to listen to a distracting tape while adding the pairs of numbers in the test phase of the experiment. The distraction should interfere with conscious retrieval, and, thus, it should diminish priming if it depends on memory strategies.

\section{Method}

Subjects. Seventy-two undergraduates at University College London participated as subjects in return for payment. They were native English speakers.

Materials. Numbers were presented as Arabic digits or dot configurations. A line divided the top from the bottom addend to segregate the two addends (see Figure 3). The number sets of Experiment 1 were altered so that each set contained two instances of each digit (see Appendix B). This was done to eliminate any influence of differences in encoding difficulty for different dot configurations. ${ }^{2}$ Number sets were assigned to study conditions (match, mismatch, new) so that each set appeared in each condition an equal number of times across subjects.

Design and Procedure. The design and procedure for Experiment 2 differed from Experiment 1 in a few respects. There was only one study phase and one test phase; the larger addend always appeared above the smaller addend. All number pairs in the study phase were presented for $1 \mathrm{sec}$ instead of remaining on the screen until the response was given. This change was not made in the test phase, in which numbers remained on the screen until the response.

A distractor task was introduced. Half the subjects were required to listen to a tape of a poem ("The Naming of Cats" by T. S. Eliot) during the test phase. They were instructed to concentrate on the tape and to try to do the addition task somewhat automatically. At the end of the experiment, they were given a short multiple-choice test about the poem consisting of five questions with three choices each.

Before beginning the experiment, the subjects performed 24 practice trials in addition to those presented in Experiment 1 , in which they named the number of dots presented on the screen. One dot configuration was presented at a time.

\section{Results and Discussion}

Incorrect responses and responses with latencies more than 2.5SDs from the mean of each subject were removed from the analysis. This resulted in a loss of $5 \%$ of responses for the subjects in both the test digit group (errors, $1 \%$; outliers, $4 \%$ ) and the test dot group (errors, $2 \%$; outliers, $3 \%$ ). For each subject, outliers were replaced by their mean plus 2.5 times their $S D$.

The results are shown in Table 2. A 2 (test notation: dot configuration, Arabic digit) $\times 3$ (study: match, mismatch, new) $\times 2$ (distractor: none, poem) mixed-design ANOVA was performed on mean RTs, with test notation and distractor as between-subjects factors. There was a main effect of test notation $\left[F(1,68)=28.55, M S_{\mathrm{e}}=\right.$ $66,545.82]$, indicating that numbers presented as dot configurations took longer to sum than did numbers presented as Arabic digits (dot configurations, $913 \mathrm{msec}$; Arabic digits, $725 \mathrm{msec}$ ). The effect of distractor was marginally significant $\left[F(1,68)=2.79, M S_{\mathrm{e}}=66,545.82, p=\right.$ $.10]$ in the predicted direction (none, $790 \mathrm{msec}$; poem, $848 \mathrm{msec})$. It did not interact with any other variable [distractor $\times$ test notation, $F(1,68)=0.08, M S_{\mathrm{e}}=$ 
Table 2

Mean Reaction Times and Their Standard Errors (in Milliseconds) and Error Proportions as a Function of Test Notation Group and Study in Experiment 2 Test Notation Group

\begin{tabular}{|c|c|c|c|c|c|c|}
\hline \multirow[b]{3}{*}{ Study } & \multicolumn{3}{|c|}{ Digit } & \multicolumn{3}{|c|}{ Dot } \\
\hline & \multicolumn{2}{|c|}{ RT } & \multirow[b]{2}{*}{ Errors } & \multicolumn{2}{|c|}{ RT } & \multirow[b]{2}{*}{ Errors } \\
\hline & $M$ & $S E$ & & $M$ & $S E$ & \\
\hline Match & 702 & 24.38 & .02 & 863 & 27.45 & .01 \\
\hline Mismatch & 714 & 21.26 & .01 & 906 & 31.75 & .02 \\
\hline New & 759 & 23.83 & .01 & 969 & 31.33 & .04 \\
\hline
\end{tabular}

66,545.82; distractor $\times$ study, $F(2,136)=0.55, M S_{\mathrm{e}}=$ $5,650.21$; distractor $\times$ study $\times$ test notation, $F(2,136)=$ $\left.0.02, M S_{\mathrm{e}}=5,650.21\right]$. Thus, the distractor task had no influence on priming. Average performance on the multiple-choice test was $81 \%$ correct, indicating that the subjects did process the information presented in the distractor task.

There was a main effect of study $[F(2,136)=21.94$, $\left.M S_{\mathrm{e}}=5,650.21\right]$ and a trend for an interaction between study and test notation $\left[F(1,136)=2.01, M S_{\mathrm{e}}=5,650.21\right.$, $p=.14]$. To further investigate the effect of study, separate planned comparisons were performed for the two test notation groups. For the test dot group, all study conditions were found to differ significantly [match vs. mismatch, $F(1,35)=6.51, M S_{\mathrm{e}}=5,181.56$; match vs. new, $F(1,35)=$ $23.15, M S_{\mathrm{e}}=8,804.43$; mismatch vs. new, $F(1,35)=$ 14.43, $\left.M S_{\mathrm{e}}=4,965.67\right]$, whereas for the test digit group, there was no significant difference between the match and mismatch conditions [match vs. mismatch, $F(1,35)=0.42$, $M S_{\mathrm{e}}=6,016.63$; match vs. new, $F(1,35)=17.13, M S_{\mathrm{e}}=$ $3,385.49$; mismatch vs. new, $F(1,35)=6.53, M S_{\mathrm{e}}=$ $5,547.49]$. Thus, only the subjects tested with dot configurations demonstrated a surface-form effect, benefiting most from exact repetition.

The results of Experiment 2 replicate the findings of Experiment 1. Facilitation was present across notations, and a surface-form effect was found only for the less typical notation in the context of the addition task. The results of Experiments 1 and 2 show that facilitation tested for Arabic digits was unaffected by the notation of the prime for both analogical (dot configurations) and discrete (Arabic digits and number words) primes. The distractor task used in Experiment 2 had no effect on priming; thus, the possibility that priming depended on consciously directed memory retrieval is unlikely. This issue will be addressed again in Experiment 4, which tested recognition memory directly.

The surface-form effects found in Experiments 1 and 2 could be taken to show that encoding processes for number words and dot configurations were facilitated in the addition task. This would be consistent with common-code models in which encoding processes, which differ for different notations, are independent of the arithmetical operation. Therefore, Experiment 3 was performed to deter- mine whether priming would occur for the same numbers presented in different arithmetical operations.

\section{EXPERIMENT 3}

In Experiment 3, notation was maintained across repetitions; however, for one third of the items, the operation performed on the prime was multiplication instead of addition. One third of the items were not presented in the study task, and the remaining third were presented for addition. As before, the test task was addition. If the surfaceform effects found in Experiments 1 and 2 reflect facilitation of encoding processes, then priming should be found for the same numbers in different operations, for number words and dot configurations, but not for Arabic digits.

\section{Method}

Subjects. Thirty-six native English-speaking undergraduates at University College London participated as subjects in return for payment.

Materials. Numbers were presented as Arabic digits, number words, or dot configurations to different groups of subjects. The number sets for the Arabic-digit and word groups were the same as those used in Experiment 1 (see Appendix A). The number sets for the dot group were the same as those used in Experiment 2 (see Appendix B). Number sets were assigned to study task conditions (add, multiply, new) so that each pair of numbers appeared equally often in each condition across subjects.

Design and Procedure. There were three groups of subjects: the Arabic-digit group, the dot group, and the word group, depending on the notation. For each group, notation was maintained across study and test phases. The subjects were randomly assigned to a notation group.

The procedure of Experiment 3 was identical to that of Experiment 2 except that items in the study phase were presented either for addition or for multiplication, and there was no distractor task during the test phase. Each study trial consisted of the following sequence of events: A rectangle containing an arithmetical sign, either for addition $(+)$ or for multiplication $(X)$, was displayed in the center of the screen for $750 \mathrm{msec}$. The sign was cleared from the screen, and the number pair appeared inside the rectangle and remained on the screen for $1 \mathrm{sec}$. (For dot configurations, a line divided the top from the bottom addend; see Figure 3.) The test trials were the same as the test trials in Experiments 1 and 2.

\section{Results and Discussion}

The results of Experiment 3 are shown in Table 3. Incorrect responses and responses with latencies more than $2.5 S D$ s from the mean of each subject were removed from the analysis. This resulted in elimination of $7 \%$ of responses (outliers, 3\%; errors, $4 \%$ ). For each subject, the outliers were replaced by their mean plus 2.5 times their $S D$.

A 3 (study task: add, multiply, new) $\times 3$ (notation: digit, word, dot) mixed-design ANOVA was performed on mean RTs, with notation as a between-subjects factor. There was a main effect of notation $[F(2,33)=34.99$, $\left.M S_{\mathrm{e}}=42,388.26\right]$. As before, Arabic digits produced the lowest RTs (Arabic digits, $669 \mathrm{msec}$; number words, $1,075 \mathrm{msec}$; dot configurations, $887 \mathrm{msec}$ ). 
Table 3

\begin{tabular}{|c|c|c|c|c|c|c|c|c|c|c|c|c|}
\hline \multirow[b]{4}{*}{ Study Task } & \multicolumn{9}{|c|}{ Notation Group } & \multirow{2}{*}{\multicolumn{3}{|c|}{ Mean }} \\
\hline & \multicolumn{3}{|c|}{ Digit } & \multicolumn{3}{|c|}{ Word } & \multicolumn{3}{|c|}{ Dot } & & & \\
\hline & \multicolumn{2}{|c|}{ RT } & \multirow[b]{2}{*}{ Errors } & \multicolumn{2}{|c|}{ RT } & \multirow[b]{2}{*}{ Errors } & \multicolumn{2}{|c|}{ RT } & \multirow[b]{2}{*}{ Errors } & \multicolumn{2}{|c|}{ RT } & \multirow[b]{2}{*}{ Errors } \\
\hline & $M$ & $S E$ & & $M$ & $S E$ & & $M$ & $S E$ & & $M$ & $S E$ & \\
\hline Add & 640 & 30.38 & .00 & 1,003 & 35.45 & .03 & 835 & 28.13 & .03 & 826 & 30.66 & .02 \\
\hline Multiply & 671 & 31.38 & .03 & 1,092 & 56.28 & .05 & 899 & 42.42 & .07 & 887 & 38.36 & .05 \\
\hline New & 696 & 36.61 & .00 & 1,129 & 41.64 & .10 & 927 & 37.51 & .05 & 917 & 36.91 & .05 \\
\hline
\end{tabular}

There was also a main effect of study task $[F(2,66)=$ 13.93, $\left.M S_{\mathrm{e}}=5,637.91\right]$. Specific comparisons showed that, although there was a suggestion of priming for number pairs that had been multiplied at study, a significant priming effect was present only for number pairs that had been added at study [add vs. new, $F(1,33)=$ $54.80, M S_{\mathrm{e}}=2,761.20$; multiply vs. new, $F(1,33)=2.51$, $M S_{\mathrm{e}}=6,601.37, p=.12$; add vs. multiply, $F(1,33)=8.96$, $\left.M S_{\mathrm{e}}=7,551.14\right]$. There was no interaction between the two factors $\left[F(4,66)=0.74, M S_{\mathrm{e}}=5,637.91\right.$, n.s. $]$, and the trend for priming across operations was present for all notations. To confirm this conclusion, separate comparisons were performed for each notation group. Three onetailed $t$ tests, comparing number pairs that had been multiplied with new pairs, produced a significant priming effect only for the digit group [digit, $t(1,11)=1.84, p=$ .05 ; word, $t(1,11)=0.85, p=.21 ; \operatorname{dot}, t(1,11)=0.82, p=$ .21]. Thus, a cross-operation priming effect did not require an atypical number notation, and it could have resulted from automatic activation of addition information at study even when the study task was multiplication. Automatic activation has been found in other paradigms in which activation of the sum of a pair of numbers is found even when the task does not demand addition but, instead, a decision on whether a probe number is one of the number pair (LeFevre et al., 1988). The probe is rejected more slowly if it is the sum of the numbers. In the present case, however, activation of addition information probably resulted from the interleaving of addition and multiplication within the same block of trials rather than from activation that might occur in the absence of any calculation task.

The results of Experiment 3 do not provide support for an interpretation of the surface-form effects found in Experiments 1 and 2 in terms of facilitation of encoding processes common to different arithmetical tasks. Surfaceform effects were found only for atypical notations in Experiments 1 and 2; in Experiment 3, however, priming for the same numbers in different arithmetical operations, which should have included the same encoding component, was independent of notation and, if anything, more reliable for Arabic digits. Thus, the pattern of results across Experiments 1, 2, and 3 is not consistent with models that do not allow form-specific representation of arithmetical information and that explain effects of notation in terms of independent encoding processes. Specifically, these results do not confirm the perceptual encoding interpretation put forward by Rickard and Bourne (1996) to explain their finding that, after extended training on multiplication problems, performance was better when the test notation matched the training notation. They offer a "less parsimonious" alternative to the encoding explanation, which also cannot capture the present results, because it explains effects of notation in terms of asemantic and, therefore, nonarithmetical information:

One possibility is that an abstract representation mediates number-fact retrieval in most everyday circumstances, but that more direct connections bypassing this stage may develop under conditions of extensive practice on a small set of problems that are always encountered in the same input format and with the same output requirements. This dualroute approach (with obvious similarities to well-known reading models) lacks parsimony, but it does account naturally both for our findings that suggest access to an abstract representation, as well as for those that suggest more direct, or asemantic, connections. (Rickard \& Bourne, 1996, p. 1293)

The findings of Experiments 1, 2, and 3 can be explained by an approach that accepts both a common code and codes that are form-specific. These form-specific codes are not assumed to reflect the stable representation of arithmetical facts, because they are particular associations established during the experimental session. Conditions in which particular items appear novel or atypical, such as number words and dot configurations presented for addition, will be the most likely to lead to form-specific effects, because the associations will be more distinctive. This interpretation differs from the asemantic interpretation quoted above (Rickard \& Bourne, 1996), because the associations are assumed to represent both notation and arithmetical information and thus form a perceptualconceptual package (Jacoby et al., 1992).

Experiment 4 was performed to determine whether notation in the addition task has consequences for a direct measure of retention when subjects must purposefully retrieve information from memory. Direct and indirect measures are often found to dissociate (Tulving, Schacter, \& Stark, 1982), but some variables have the same effects on both measures. Both measures are enhanced when the study and test lists share a high proportion of items and 
when the study-test interval is short (Jacoby, 1983). Typicality may be another variable that similarly affects direct and indirect measures. If conditions that showed high levels of priming in Experiments 1 and 2 also show high levels of recognition memory, this would be problematic for the conclusion from Experiment 2 that priming did not depend on conscious retrieval.

Recall of notation has been shown to be better for number words than for Arabic digits, but only for numbers above 10 and only when the study task involved copying the numbers (Vaid \& Frenck-Mestre, 1991). However, when the study task requires addition, even single-digit numbers may produce higher levels of retention if they are presented in atypical notations. In the verbal domain, Kolers $(1973,1975)$, for example, showed that inverted sentences were more likely to be recognized as having been seen before than were normally presented sentences. In the recognition test, subjects, who were required to categorize sentences into new, same-form, and differentform categories, showed higher levels of retention for sentences that were inverted at study and test than for sentences that were normally presented at study and test. Therefore, Experiment 4 tested the effect of notation on recognition memory: Subjects were simply asked to judge whether or not they had added pairs of numbers in a prior addition study task.

\section{EXPERIMENT 4}

\section{Method}

Subjects. Twenty-four undergraduates at University College London participated in return for payment. Twelve native Italian speakers, on a student exchange program, participated as one group, and 12 native English speakers participated as a second group.

Materials. For the Italian group, numbers were presented as Arabic digits and Italian words; for the English group, numbers were presented as Arabic digits and dot configurations. The number pairs for the Italian group were the same as those used in Experiment 1 (always presented with the larger addend above the smaller addend), and the number pairs for the English group were the same as those used in Experiment 2. Number sets were assigned to study conditions so that each set appeared an equal number of times in each condition.

Design and Procedure. The English subjects were randomly assigned to either a test Arabic group or a test dot group. The Italian subjects were randomly assigned to either a test Arabic group or a test word group. The design and procedure of Experiment 4 were identical to those of Experiment 2 except that there was no distractor task, and the test phase consisted of a recognition memory test. For each pair of numbers presented in the test phase, the subjects indicated whether they had added them in the addition block, in any notation, by saying "old" if they thought that they had and "new" if they thought that they had not. Each pair of numbers was displayed for 1 sec. After a 750-msec blank interval, a prompt appeared on the screen, and the subjects were required to respond if they had not already done so. After the experimenter entered the response on the keyboard, the next trial began.

\section{Results and Discussion}

The results are shown in Figure 4. Analyses were carried out on the results of Experiment 4 considering the data from the non-Arabic conditions of the two groups as a level of the variable test notation. The overall probability of a false alarm ("old" responses to new items) was .31, and this did not differ significantly across conditions. A 2 (group: Italian-word, English-dot) $\times 2$ (test notation: Arabic, non-Arabic) ANOVA was performed on false alarms, with the variables of group and test notation analyzed as between-subjects factors. There was no effect of test notation $\left[F(1,20)=0.82, M S_{\mathrm{e}}=0.05\right.$, n.s. $]$ and no test notation $\times$ group interaction $\left[F(1,20)=0.03, M S_{e}=\right.$ $0.05, \mathrm{n.s.]}$. However, there was a trend for fewer false alarms for the English group (.23) than for the Italian group $(.38)\left[F(1,20)=2.65, M S_{\mathrm{e}}=0.05, p=.12\right] .^{3}$

The main analysis was performed on recognition scores, calculated for each subject by subtracting their falsealarm rate from their hit rate for each condition. A 2 (group: Italian-word, English-dot) $\times 2$ (test notation: Arabic, non-Arabic) $\times 2$ (study notation: Arabic, nonArabic) mixed-design ANOVA, with group and test notation as between-subjects factors, revealed a main effect of group $\left[F(1,20)=4.16, M S_{\mathrm{e}}=0.12\right]$, but this variable did not interact with any other variable [group $\times$ study notation, $F(1,20)=0.02, M S_{\mathrm{e}}=0.04$, n.s.; group $\times$ test notation, $F(1,20)=0.06, M S_{\mathrm{e}}=0.04$, n.s.; group $\times$ study notation $\times$ test notation, $F(1,20)=0.17, M S_{\mathrm{e}}=0.04$, n.s.]. There was a marginally significant effect of test notation $\left[F(1,20)=2.94, M S_{\mathrm{e}}=0.12, p=.10\right]$, a significant effect of study notation $\left[F(1,20)=9.91, M S_{\mathrm{e}}=0.04\right]$, and a study notation $\times$ test notation interaction $[F(1,20)=$ 5.41, $\left.M S_{\mathrm{e}}=0.04\right]$. Recognition scores (hit rate - falsealarm rate) were higher for the subjects tested with Arabic digits than for the subjects tested with non-Arabic notations, but addition performed on non-Arabic notations at study produced higher recognition scores than did addition performed on Arabic digits at study. As can be seen in Figure 4, these differences seem to depend on relatively low scores for addition performed on Arabic digits tested with non-Arabic notations.

The above analysis was confirmed by an analysis of $d^{\prime}$ scores from signal detection theory (see Figure 4 for mean $d^{\prime}$ prime scores) [main effect of group, $F(1,20)=2.03$, $M S_{\mathrm{e}}=0.50 ;$ group $\times$ study notation, $F(1,20)=0.02$, $M S_{\mathrm{e}}=0.18$, n.s.; group $\times$ test notation, $F(1,20)=0.01$, $M S_{\mathrm{e}}=0.50$, n.s.; group $\times$ study notation $\times$ test notation, $F(1,20)=0.20, M S_{\mathrm{e}}=0.18$, n.s.; test notation, $F(1,20)=$ $3.46, M S_{\mathrm{e}}=0.50, p=.08$; study notation, $F(1,20)=5.03$, $M S_{\mathrm{e}}=0.18$; study notation $\times$ test notation, $F(1,20)=$ 9.82, $\left.M S_{\mathrm{e}}=0.18\right]$.

To determine whether numbers presented in atypical notations at study and test were better recognized than numbers presented in Arabic digit notation at study and test, analogous to Kolers's $(1973,1975)$ results for normal and inverted text, a comparison was carried out on recognition scores. No significant difference was found [Arabic/ Arabic $=.42 ;$ non-Arabic $/$ non-Arabic $=.43 ; t(1,22)=0.12$, n.s.]. The effect might have been absent because, unlike in Kolers's studies, the subjects were not required to decide whether surface form matched at study and test. Furthermore, the variable of test notation was manipulated be- 
Italian group

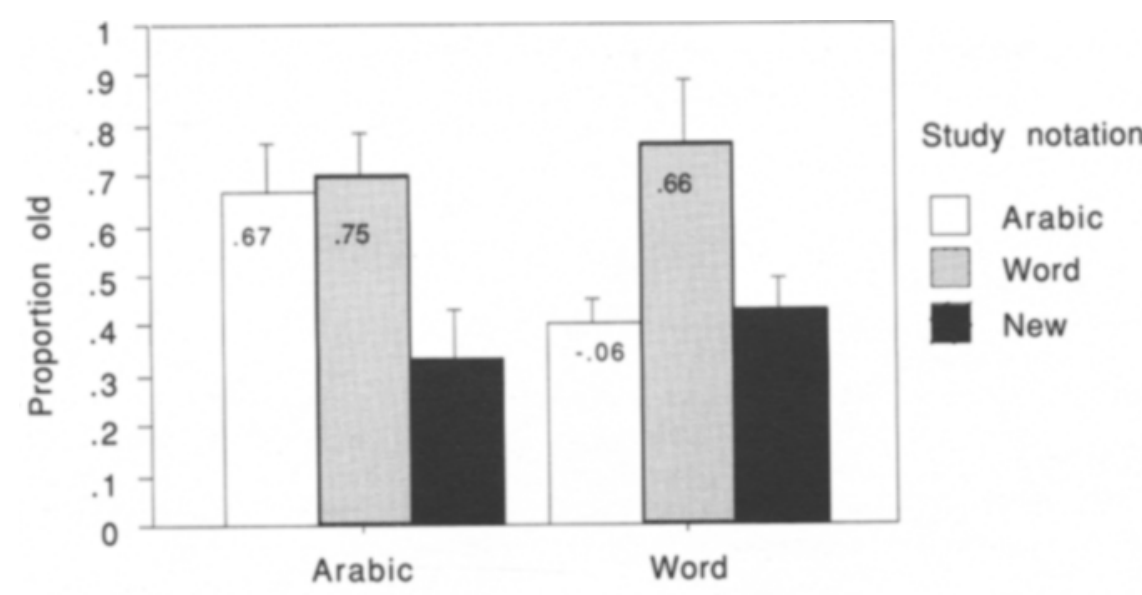

Test Notation

English group

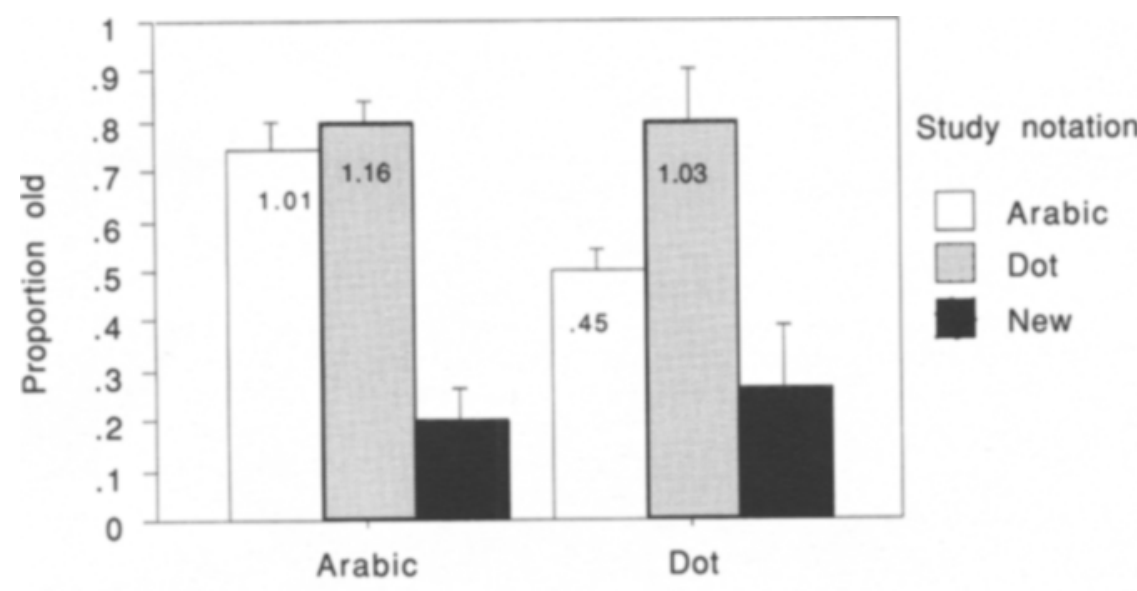

Test Notation

Figure 4. Recognition memory (proportion judged old) in Experiment 4 as a function of group, study notation, and test notation. (T-bars indicate standard errors.) Mean $d^{\prime}$ scores are given, inside each bar, for each of the repeated conditions.

tween subjects in the present experiment so that any effects that might depend on perceived differences in typicality at test would be absent in this experiment.

To further investigate the study notation $\times$ test notation interaction, an analysis of simple effects was performed on recognition scores. This showed that the effect of test notation was significant for items that were presented as Arabic digits at study, but not for items presented in nonArabic notations [Arabic, $F(1,20)=6.20, M S_{\mathrm{e}}=0.10$; non-Arabic, $F(1,20)=0.09, M S_{\mathrm{e}}=0.07$, n.s.]. Hence, recognition memory for Arabic digits was better when it was tested with Arabic digits than when it was tested with non-Arabic notations. This does replicate a result found by Kolers $(1973,1975)$, in which recognition of a sentence printed in normal type was better when it reappeared in normal type than when it reappeared inverted.

The final analysis of simple effects showed that the effect of study notation was significant only for numbers presented in non-Arabic notations at test [Arabic, $F(1,20)=$ $0.34, M S_{\mathrm{e}}=0.04$, n.s.; non-Arabic, $F(1,20)=14.98, M S_{\mathrm{e}}=$ 0.04]. Therefore, as for the priming measure in Experiments 1 and 2, when the test items were Arabic digits, 
there was no significant difference between different study notations.

The results of Experiment 4 can be summarized as follows: Overall, recognition memory for number pairs studied in non-Arabic notations was better than memory for number pairs studied in Arabic digit notation, because performance for Arabic digits when non-Arabic notations were used in the recognition memory test was poor, relative to all the other conditions. This could have occurred because the subjects, unable to ignore the surfaceform information contained in the atypical non-Arabic notations, misattributed a general sense of unfamiliarity for the number pairs previously seen as Arabic digits to not having seen them at all.

The fact that recognition tested with Arabic digits showed no sensitivity to study surface form suggests that the subjects were able to contact abstract or conceptual addition information in the time allowed. In this sense, the overall pattern of data mirrors the priming results of $\mathrm{Ex}$ periments 1 and 2 , in which performance was unaffected by the study notation when addition was tested with Arabic digits. However, because recognition, unlike priming, was relatively low for Arabic digits repeated later as words or dot configurations, the results of Experiment 4 pose no difficulty for the conclusion that priming in Experiments 1 and 2 did not result from conscious memory strategies.

\section{GENERAL DISCUSSION}

A characteristic pattern of asymmetric surface-form effects was found in Experiments 1 and 2. Surface-form effects were present only for atypical notations, a pattern also found in the verbal priming literature (Brown \& Carr, 1993). Notation also influenced recognition memory in Experiment 4. The indirect measure (priming) and the direct measure (recognition) produced parallel results in the sense that re-presentation of an identical, rather than a visually distinct, number pair resulted in better performance when retention was tested with atypical notations. The speeded recognition task may have recruited a retrieval process that, for the atypical notations, did not heavily weight conceptual information. Thus, the implications of these experiments are not that the same processes function in the priming and recognition tasks, but that the notations that were atypical in the context of addition had analogous consequences. The importance of typicality is supported by the finding that, for priming and recognition memory, the same asymmetric pattern of results was found when Arabic digits were presented with number words and when Arabic digits were presented with dot configurations - two very different notations that share only the characteristic that they are not normally used in calculation. The simplest interpretation of these findings, and similar findings in other domains, is that atypical surface forms require processing that focuses attention on perceptual features of the display and that therefore influence representation. This can be seen as the cognitive system adapting to novel demands. For number words and dot configurations, surface-form effects in priming may reflect tuning to these notations that are not normally used in calculation.

The common-code models of numerical processing do not explicitly take into account changes that might occur as a result of notations that are atypical for arithmetical tasks. Although these models assume that encoding processes differ for different notations, the results of Experiment 3 are not consistent with the pure encoding interpretation they offer. If the differences in priming for typical and atypical notations found in Experiments 1 and 2 were attributable to encoding processes specific to particular notations but independent of arithmetical operations, these processes should have been isolated by Experiment 3 when the same numbers were presented in the same notations but in different operations. Experiments 1 and 2 might be taken to suggest that the encoding stage for the atypical notation required more time than the encoding stage for the typical notation. However, Experiment 3 showed that the time required for the postulated encoding processes for typical and atypical notations did not differ, because priming for the same numbers presented in different tasks did not depend on notation. Thus, common-code models that explain surface-form effects in terms of encoding processes that depend on the input notation, but not on the particular arithmetical operation to be performed, are not consistent with the present results.

The preferred-entry code model seems to directly predict the asymmetry between typical and atypical notations, because it admits a role for different skill levels tied to particular notations. But, to the extent that this model shares the assumption of the other common-code models that the input notation is not represented once encoding has taken place, the model is also not supported.

Finally, even the encoding complex hypothesis is not entirely compatible with the present results, because performance tested for the Arabic-digit notation did not demonstrate any surface-form effects. The general motivation of this model to challenge abstractionist approaches is not supported by the present data. Furthermore, the surface-form effects for number words and dot configurations are unlikely to be accountable in terms of notation-specific codes in a semantic memory network, because the subjects had probably rarely, if ever, experienced these notations in calculation tasks before the experimental session.

The alternative suggested by these experiments-that numerical information is represented in terms of both a common code and form-specific codes-is consistent with a variety of other findings. For example, form-specific representation has been demonstrated by on-line processing differences for different notations (Blankenburger \& Vorberg, 1997; Noel \& Seron, 1997). Similar to the present findings, other results from a training paradigm that were taken to support a pure common-code approach have been acknowledged to be consistent with both a commoncode and asemantic form-specific representation (Rickard \& Bourne, 1996). According to the present proposal, however, the form-specific representations are associations 
formed during the experimental session that code both notation and semantic (arithmetical) information.

Thus, the final conclusion may not seem to clearly distinguish between the alternatives put forward, since both abstract and form-specific systems are needed. It is clear, however, that the surface-form effects reported in this study depended on the novelty of the stimuli as input for the task.

\section{REFERENCES}

ARguthnotT, K. D. (1996). To repeat or not to repeat: Repetition facilitation and inhibition in sequential retrieval. Journal of Experimental Psychology: General, 125, 261-283.

AshCRAFT, M. H. (1992). Cognitive arithmetic: A review of the data and theory. Cognition, 44, 75-I 06.

Blankenburger, S., \& VorberG, D. (1997). The single-format assumption in arithmetical fact retrieval. Journal of Experimental Psychology: Learning, Memory, \& Cognition, 23, 721-738.

BROwN, J. S., \& CARR, T. H. (1993). Limits on perceptual abstraction in reading: Asymmetric transfer between surface forms differing in typicality. Journal of Experimental Psychology: Learning, Memory, \& Cognition, 19, 1277-1296.

Campbell, J. I. D. (1994), Architecture for numerical cognition. Cognition, 53, 1-44.

CAMPBELL, J. I. D. (1995). Mechanisms of simple addition and multiplication: A modified network-interference theory and simulation. Mathematical Cognition, 1, 121-164.

CLARK, J. M., \& CAMPBELL, J. I. D. (1991). Integrated versus modular theories of number skills and acalculia. Brain \& Cognition, 17, 204-239.

DeHAENE, S. (1992). Varieties of numerical abilities. Cognition, 44, 1-42.

DEHAENE, S., \& COHEN, L. (1995). Towards an anatomical and functional model of number processing. Mathematical Cognition, 1, 83-120.

Gonzalez, E. G., \& Kolers, P. A. (1982). Mental manipulation of arithmetic symbols. Journal of Experimental Psychology: Learning, Memory, \& Cognition, 8, 308-319.

Groen, G. J., \& Parkman, J. M. (1972). A chronometric analysis of simple arithmetic. Psychological Review, 79, 329-343.

JACOBY, L. L. (1983). Perceptual enhancement: Persistent effects of an experience. Journal of Experimental Psychology: Learning, Memory, \& Cognition, 9, 21-38.

JACOBY, L. L. (1991). A process dissociation framework: Separating automatic from intentional uses of memory. Journal of Memory \& Language, 30, 513-541.

JACOBY, L. L., \& HAYMAN, C. A. G. (1987). Specific visual transfer in word identification. Journal of Experimental Psychology: Learning, Memory, \& Cognition, 13, 456-463.

JaCOBY, L. L., Levy B. A., \& SteinBaCh, K. (1992). Episodic transfer and automaticity: Integration of data-driven and conceptuallydriven processing in rereading. Journal of Experimental Psychology: Learning, Memory, \& Cognition, 18, 15-24.

Kolers, P. A. (1973). Remembering operations. Memory \& Cognition, 1, 347-355.

KOLERS, P. A. (1975). Memorial consequences of automatized encoding. Journal of Experimental Psychology: Human Learning \& Memory, 1 , 689-701.
LeFevre, J., Bisanz, J., \& MrkonjIC, L. (1988). Cognitive arithmetic: Evidence for obligatory activation of arithmetic facts. Memory \& Cognition, 16, 45-53.

LeFevre, J., Sadesky, G. S., \& Bisanz, J. (1996). Selection of procedures in mental addition: Reassessing the problem size effect in Adults. Journal of Experimental Psychology: Learning, Memory \& Cognition, 22, 216-230.

Marsolek, C. J., Kosslyn, S. M., \& Squire, L. (1992). Form-specific visual priming in the right cerebral hemisphere. Journal of Experimental Psychology: Learning, Memory, \& Cognition, 18, 492-508.

McCloskey, M., Caramazza, A., \& Basili, A. (1985). Cognitive mechanisms in number processing and calculation: Evidence from dyscalculia. Brain \& Cognition, 4, 171-196.

NOEL, M. P., \& SERON, X. (1992). Notational constraints and number processing: A reappraisal of the Gonzalez and Kolers (1982) study. Quarterly Journal of Experimental Psychology, 45A, 451-478.

NoEl, M. P., \& SERON, X. (1993). Arabic number reading deficit: A single case study. Cognitive Neuropsychology, 10, 317-339.

NoEL, M. P., \& SERON, X. (1997). On the existence of intermediate representations in numerical processing. Journal of Experimental Psychology: Learning, Memory, \& Cognition, 23, 697-720.

RiCKARD, T. C., \& BouRnE, L. E. (1996). Some tests of an identical elements model of basic arithmetic. Journal of Experimental Psychology: Learning, Memory, \& Cognition, 22, 1281-1295.

Rickard, T. C., Healy, A. F., \& Bourne, L. E. (1994). On the cognitive structure of basic arithmetic skills: Operation, order, and symbol transfer effects. Journal of Experimental Psychology: Learning. Memory, \& Cognition, 20, 1139-1153.

RoEdiger, H. L., III, \& BLAXTON, T. A. (1987). Effects of varying modality, surface features, and retention interval on priming in wordfragment completion. Memory \& Cognition, 15, 379-388.

Schacter, D. L., Chiv, C. Y. P., \& Ochsner, K. N. (1993). Implicit memory: A selective review. Annual Review of Neuroscience, 16, 159-182.

Tulving, E., Schacter, D. L., \& Stark, H. A. (1982). Priming effects in word-fragment completion are independent of recognition memory. Journal of Experimental Psychology: Learning. Memory, \& Cognition, 8, 336-342.

VAID, J., \& FRENCK-MESTRE, C. A. (1991). Incidental memory for format of presentation of number stimuli: Evidence from monolinguals and bilinguals. Brain \& Cognition, 17, 272-284.

ZBrodoff, N. J., \& Logan, G. D. (1986). On the autonomy of mental processes: A case study of arithmetic. Journal of Experimental Psychology: General, 115, 118-130.

ZHANG, J., \& NORMAN, D. A. (1995). A representational analysis of numeration systems. Cognition, 57, 271-295.

\section{NOTES}

1. Unless otherwise stated $p<.05$.

2. We would like to thank Michael McCloskey for suggesting that we should control for encoding difficulty.

3. This could have occurred because, for the English group, one set of numbers (see Appendix B) contained four pairs whose sums differed from the sums of the pairs contained in the other two sets. Therefore, when these items were new, they could be more easily detected as such. 
APPENDIX A

\begin{tabular}{cccc}
\hline & $\begin{array}{c}\text { Large } \\
\text { Addend }\end{array}$ & $\begin{array}{c}\text { Small } \\
\text { Addend }\end{array}$ & Sum \\
\hline 1 & 4 & 1 & 5 \\
& 6 & 1 & 7 \\
5 & 3 & 8 \\
& 6 & 3 & 9 \\
& 7 & 3 & 10 \\
2 & 3 & 2 & 5 \\
& 5 & 2 & 7 \\
& 7 & 1 & 8 \\
& 5 & 4 & 9 \\
& 8 & 2 & 10 \\
3 & 3 & 1 & 4 \\
& 4 & 3 & 7 \\
& 6 & 2 & 8 \\
& 7 & 2 & 10 \\
\hline
\end{tabular}

APPENDIX B

\begin{tabular}{cccc}
\hline & $\begin{array}{c}\text { Large } \\
\text { Addend }\end{array}$ & $\begin{array}{c}\text { Smail } \\
\text { Addend }\end{array}$ & Sum \\
\hline 1 & 3 & 2 & 5 \\
& 5 & 2 & 7 \\
& 4 & 4 & 8 \\
& 6 & 3 & 9 \\
& 6 & 5 & 11 \\
2 & 3 & 3 & 6 \\
& 4 & 2 & 6 \\
& 6 & 2 & 8 \\
& 6 & 4 & 10 \\
& 5 & 5 & 10 \\
3 & 2 & 2 & 4 \\
& 4 & 3 & 7 \\
& 5 & 3 & 9 \\
& 5 & 4 & 12 \\
\hline
\end{tabular}

(Manuscript received August 27, 1997 ;

revision accepted for publication February 27, 1998.) 